ROCZNIKI TEOLOGICZNE

Tom LXVI, zeszyt 7 - 2019

DOI: https://dx.doi.org/10.18290/rt.2019.66.7-5

PÉTER SZENTPÉTERY

\title{
WELTETHOS, NEUER ATHEISMUS UND CHRISTLICHE IDENTITÄT
}

\author{
GLOBAL ETHIC (WELTETHOS), NEW ATHEISM \\ AND CHRISTIAN IDENTITY
}

\begin{abstract}
A b s tra ct. The Global Ethic Project is closely connected to the name of its founder, Hans Küng (1928). It is based on the fact that in different religions there are similar commandments which all can be summed up by the Golden Rule. The first part introduces the project itself. It was mainly criticized because it extends the western Christian notion of humanity to other religions, too. This is dealt with in the second part. Hans Küng wants to involve not only the believers but also the atheists, agnostics etc. because Global Ethic is primarily of ethical character. That is why the author focuses on two high-impact works of two prominent representatives of the so-called new atheists in quest for an answer to the question to what extent they fit in with the Project. $\mathrm{He}$ finally sums up the conclusions in eight theses.
\end{abstract}

Key words: Global Ethic; Hans Küng; religion; dialogue; humanity; New Atheism; Stephen Hawking; Richard Dawkins.

\section{PROJEKT WELTETHOS — GEMEINSAME PRINZIPIEN}

Hans Küng hatte schon die Grundlagen in seinem Buch Christentum und Weltreligionen im Jahre 1984 gelegt. ${ }^{1}$ Im Februar 1989 hatte er einen

Dr. PÉTER SzentPÉTERY - Privatdozent an der Evangelisch-Lutherischen Theologischen Universität (Kirchliche Hochschule) in Budapest. Er unterrichtet Konfessionskunde/Ökumene, Religionsgeschichte, Weltreligionen und beschäftigt sich mit Fragen von Glauben und Wissen, in seinem Habilitationsbuch mit Darwins Wirkung auf den Schöpfungsglauben (2008). Er war zwischen 2000-2015 Sekretär des Ökumenischen Studienzentrums, Budapest und 2012-2014 Sekretär der Societas Oecumenica; Korrespondenzadresse - E-Mail: peter.szentpetery@lutheran.hu.

${ }^{1}$ Hans KÜNG, Christentum und Weltreligionen (München: Herder, 1984). Schon in Christ sein (1974) wird den Weltreligionen ein Kapitel gewidmet (A III). Hier wird vor allem auf die Suche nach der Wahrheit seitens der anderen Religionen und auf die Notwendigkeit der Selbstkritik seitens des Christentums hingewiesen. Allgemeine überlappende ethische Grundprinzipien sind kaum unterstrichen. Hans KüNG, Christ sein (München: Dt. Taschenbuch-Verlag, $\left.{ }^{8} 1987\right), 97-131$. 
Vortrag an der Konferenz der UNESCO in Paris unter dem Titel „Es gibt keinen Frieden ohne Religionsfrieden“"gehalten, und im Februar 1990 beim Weltwirtschaftsforum von Davos: „Warum brauchen wir globale ethische Normen zum Überleben?" Sein zusammenfassendes und programmgebendes Werk Projekt Weltethos ist im Mai 1990 erschienen. Er hatte einen umfassenden Vortrag in Tübingen am 18. Juni gehalten, an dem ich als Stipendiat teilnehmen konnte. Im Jahre 1993 hatte das Parlament der Weltreligionen in Chicago die Allgemeine Erklärung des Weltethos verabschiedet. Die Stiftung Weltethos wurde 1995 gegründet. Aufgrund des Projekts wurde am 6. Oktober 2009 das Manifestum der Globalen Wirtschaftsethik proklamiert. ${ }^{2}$ Das Institut für Weltethos wurde am 18. April 2012 eröffnet. $^{3}$

Der Vater des Projekts stellt nachdrücklich fest: das Weltethos ist keine Einheitsreligion oder Einheitskultur. Die Religionen können nicht auf einen gemeinsamen Nenner gebracht werden und man dürfe nicht danach streben. ${ }^{4}$

Die Religionen haben gemeinsame Züge, weil sie menschliche Grundfragen beantworten möchten: Woher kommt die Welt und ihre Ordnung? Warum sind wir geboren und werden sterben? Warum gibt es sittliches Bewußtsein und sittliche Normen?

Auch diejenigen, die die Religionen zurückweisen, müssen sie ernst nehmen, also auch die sogenannten neuen Atheisten, von denen später gesprochen wird. Alle großen Religionen erfordern gewisse ethische Grundnormen, Grundprinzipien, die auf ein unbedingtes Absolutes gegründet wer-

Als Grundlage dieses Aufsatzes diente ein Workshop-Vortrag, der an der XVII. Wissenschaftlichen Konsultation der Societas Oecumenica, Belfast, 23-28. August 2012 gehalten wurde. Durchgesehen und aktualisiert im Januar 2019.

Zu Punkten 1. und 2. dieses Aufsatzes vgl. die Punkte 1. und 2. von Péter SzentPÉTERY und András SzalaI, „Világetosz, párbeszéd, egyezkedő szó“ [Weltethos, Dialog, und gemeinsames Wort], Vallástudományi Szemle [Religionswissenschaftliche Schau, Vierteljahresschrift der Ungarischen Gesellschaft für Religionswissenschaft] 2 (2011): 11-25., bes. 11-16.

${ }^{2}$ Manifesto, Global Economic Ethic, Consequencen for Global Businesses. UN Headquarters, New York, 6 October 2009, Stand: 11.01.2019, www.globaleconomicethic.org/main/pdf/ENG/ we-manifest.ENG.pdf.

${ }^{3}$ Weltethos-Institut, Stand: 11.01.2019, http://www.weltethos-institut.org/institut/.

${ }^{4}$ Hans KüNG, Projekt Weltethos (München: Piper, 1990), 149 f. — Zur Literatur s. Bibliographie zur Weltethos-Debatte, Stand: 11.01.2019, https://www.weltethos.org/1-pdf/40-literatur/ deu/bib-we-01_11.pdf. Hier seien nur vier seiner Werke zum Thema erwähnt: Hans KüNG, Spurensuche. Die Weltreligionen auf dem Weg (München, Zürich: Piper, ${ }^{4} 2000$ ) in der populären Form eines Bilderbuches. Hans KüNG (Hg.), Dokumentation zum Weltethos (München, Zürich: Piper, 2002); Hans KüNG (Hg.), Ja zum Weltethos. Perspektiven für die Suche nach Orientierung (München: Piper, 1995); Hans KüNG und Angela RINN-MAURER, Weltethos christlich verstanden. Positionen, Erfahrungen, Impulse (Freiburg, Basel, Wien: Herder, 2009). 
den, auch wenn diesen nicht immer oder oft nur mit Beschränkungen gefolgt wird. Es geht konkret um die fünf grossen Gebote der Menschlichkeit, die auch in der Gesellschaft und in der Wirtschaft zur Geltung gebracht werden müssten und müssen: Du sollst nicht töten, nicht lügen, nicht stehlen, nicht Ehe brechen und du sollst deine Eltern/Ahnen ehren. (vgl. Matth 19,18f. par.) Als Zusammenfassung dieser Gebote ist die Goldene Regel über religiöse und kulturelle Grenzen hinweg zu finden. ${ }^{5}$ Welthethos ist keine ,[...] WeltEthik im Sinne einer ganz besonderen philosophischen und theologischen Lehre von den sittlichen Haltungen, Werten und Normen, sondern meint die sittliche Grundhaltung des Menschen, individuell oder kollektiv verstanden." 6

Fügen wir hinzu: auch auf den religiösen Hintergrund von allerlei humanistischen Überzeugungen und Philosophien kann mehr oder weniger hingewiesen werden, sei es dadurch, dass sie sich gegenüber einer Religion (in der Regel dem Christentum) artikulieren. Auch die Neuen Atheisten gehen von der heutzutage „schon endgültigen“ Dethronisierung Gottes durch die Wissenschaft aus wie früher die Marxisten-Leninisten. Zwei von den drei Mottos des Projekts sprechen sowieso von Religionen, und auch das erste mehr oder weniger, wenn Religion als Merkmal nationaler Zugehörigkeit betrachtet wird:

Kein menschliches Zusammenleben ohne ein Weltethos der Nationen.

Kein Frieden unter den Nationen ohne Frieden unter den Religionen.

Kein Frieden unter den Religionen ohne Dialog unter den Religionen. ${ }^{7}$

Die obigen Prinzipien wurden vom Parlament der Weltreligionen in der Erklärung von Chicago zum Ausdruck gebracht, aus Anlass des hundertjährigen Jubiläums der ersten ähnlichen Zusammenkunft an Ort und Stelle, also im Jahre 1993. Sie beruft sich auf die Goldene Regel, die als feste, unerschütterliche Norm für Familien und Gemeinschaften, Rassen, Nationen und Religionen gestellt wird. Die vier Grundprinzipien, genauer gesagt, „unverrückbare Weisungen“:

Verpflichtung auf eine Kultur der Gewaltlosigkeit und der Ehrfurcht vor allem Leben.

Verpflichtung auf eine Kultur der Solidarität und eine gerechte Wirtschaftsordnung.

\footnotetext{
${ }^{5}$ KÜNG, Projekt Weltethos, 82 ff.

${ }^{6}$ Hans KüNG, Weltethos für Weltpolitik und Weltwirtschaft (München, Zürich: Piper, $\left.{ }^{3} 1998\right), 147$.

${ }^{7}$ KÜNG, Projekt Weltethos, 171.
} 
Verpflichtung auf eine Kultur der Toleranz und ein Leben in Wahrhaftigkeit.

Verpflichtung auf eine Kultur der Gleichberechtigung und die Partnerschaft von Mann und Frau.

An der UNO-Vollversammlung am 9. November 2001 zum Schluss des Jahres des Dialogs der Zivilisationen hat Hans Küng das Wesentliche im Weltethos zusammengefasst. Er sagte u. a.: „In unseren Tagen treten die Religionen wieder als Akteure in der Weltpolitik in Erscheinung. Es ist wahr, viel zu oft haben die Religionen im Lauf der Geschichte ihre zerstörerische Seite gezeigt. [...] Aber in vielen Fällen haben sie Verständigung, Versöhnung, Zusammenarbeit und Frieden angeregt und legitimiert.“ Die Basis des Weltethos sei vor allem das Prinzip der Menschlichkeit, das Humanum wie es in der UNO-Menschenrechtserklärung zu lesen ist: „Jeder Mensch ob Mann oder Frau, weiß oder farbig, reich oder arm, jung oder alt muss menschlich behandelt werden." Noch deutlicher werde es in der Goldenen Regel ausgedrückt: „Was du nicht willst, das man dir tu’, das füge auch keinem anderen zu. “8

\section{KRITIK - EIGENER MASSSTAB}

Die Kritik wird also auf die folgende Frage zugespitzt: Wie kann das Humanum zum Maßstab der Bewertung der Religionen gemacht werden? Man kann die ausführliche und tiefgreifende Kritik von Heinzpeter Hempelmann so zusammenfassen: Küng, während er von dem Wahrheitsanspruch der einzelnen Religionen absieht, bewertet die Religionen aufgrund des Begriffs des Humanums, der auf dem Boden der christlichen Kultur und der Aufklärung gewachsen ist, und reisst die ethischen Prinzipien aus dem ursprünglichen Zusammenhang der Religionen heraus. ${ }^{9}$ D. h., er lässt den Wahrheitsanspruch maximal ausser Acht, der im Falle jeder Religion unter-

\footnotetext{
${ }^{8}$ Hans KÜNG, „Dialog der Kulturen“, 9. November 2001, [also, wie er selber betont, nicht lange nach dem 11. September], https://www.weltethos.org/1-pdf/20-aktivitaeten/deu/politik/unstatement-Kueng.pdf . Vgl. Hans KüNG, Was ich glaube (München, Zürich: Piper, 2010), 294 ff. S. dazu Jürgen Bauschke, Die Goldene Regel. Staunen - Verstehen - Handeln (Berlin: EBVerlag, 2010), 11. Hier im Vorwort wird betont, daß die letzte wissenschaftliche Monographie zur Goldenen Regel in Deutschland 1962 erschienen ist.

${ }^{9}$ Heinzpeter HempelmanN, „Intolerante Toleranz. Hans Küngs »Projekt Weltethos« als Prokrustesbett religiöser Geltungsansprüche“, Theologische Beiträge 33, Febr. 2002, https://heinzpeter-hempelmann.de/hph/wp-content/uploads/2013/01/kueng.pdf
} 
schiedlich ist, „[...] für mich aber gibt es nur eine wahre Religion, das Christentum“. Auch die Toleranz des Hinduismus sei keine Ausnahme, weil er, wenn er die Möglichkeit der völligen Kenntnis der Wahrheit von allen anderen Religionen abstreitet, das Abstreiten zur Wahrheit macht. Auch Küng geht auf dieser Spur: „[...] selbsverständlich gibt es verschiedene wahre Religionen“. ${ }^{10}$ Auf diese Weise sagen und intendieren sie alle dasselbe. Sollte eine ,[...] Religion im Dialog [...] auf ihrem eigenen Wahrheitskriterium insistieren, wäre ein echter Dialog von vornherein aussichts-

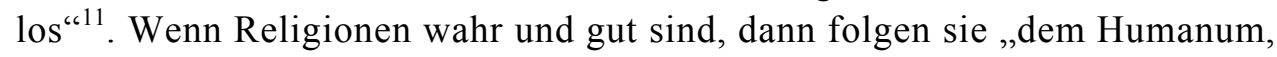
dem wahrhaft Menschlichen“, also der „Menschenwürde und den ihr zugeordneten Grundwerten“. ${ }^{12}$ Das Humanum ist also nach Hempelmanns Kritik das Wesen der absoluten Religion, die allen anderen Religionen übergeordnet ist. Hier vermittelt praktisch die biblisch-jüdisch-christliche Überlieferung unverzichtbare und womöglich neue Werte anderen Kulturen und Völkern. ${ }^{13}$ Küng vergisst aber auch nicht zu sagen, dass „Freiheit, Gleichheit, Brüderlichkeit und Menschenwürde“ ursprünglich christliche Werte waren. ${ }^{14}$ Hempelmann macht darauf aufmerksam, dass „,vergleichbare, im Sinne von inhaltlich verwandte Aussagen [...] nur der dort finden [kann], der sie aus den für sie spezifischen und ihnen ihre Bedeutung gebenden Kontexten herausreißt“. In der Kenntnis der kaum überbietbaren Theonomie des Koran wäre es schwerlich zu behaupten, dass er das Humanum zum Ziel habe. ${ }^{15}$ „Wahre Religion ist die Vollendung wahrer Menschlichkeit.“ Nach Hempelmann ist es ein Zirkelschluss: natürlich ist diese Feststellung wahr, wenn man schon im Voraus weiss, wie wahre Menschlichkeit vorzustellen ist. „Das Humanum ist eine Mindestforderung an jede Religion.“ 16

Die Kriterien für die wahre Menschlichkeit können nur vom wahren Gott festgestellt und geboten werden. Steht unser dem Christentum entstandener Humanumsbegriff ihnen nicht ganz nahe, sogar am nächsten unter allen Religionen $?^{17}$

${ }^{10}$ A.a.O., 2., 5., KÜNG, Projekt Weltethos, 129.

${ }^{11}$ HempelmanN, „Intolerante Toleranz“, 6; KüNG, Projekt Weltethos, 113.

${ }^{12}$ HempelmanN, „Intolerante Toleranz“, 7; KÜNG, Projekt Weltethos, 129.

${ }^{13}$ Hempelmann, „Intolerante Toleranz“, $7 \mathrm{f}$.

${ }^{14}$ A.a.O., 9.

${ }^{15}$ A.a.O., 11

${ }^{16}$ A.a.O. 12; KÜNG, Projekt Weltethos, 116, 121.

${ }^{17}$ Vgl. dazu Johannes ReHM, Weltethos praktisch. Perspektiven für den interreligiösen Dialog (Mainz: Matthias-Grünewald-Verlag, 2004), 64.: „Der Grund für die überraschende Übereinstimmung von Menschen verschiedener Religionsgemeinschaften in Erfahrung des guten und gelin- 
Der im vorigen Jahr verstorbene Robert Spaemann sagte schon früher Ähnliches in seiner Kritik. Nach Küng haben sich alle Ideologien ,abgewirtschaftet", und was übrigbleibt, ist das Humanum, das offenbar von jeder Ideologie frei sei, und darauf warte, ,operationalisiert“ zu werden. An dieser Operationalisierung des Humanum hänge nicht weniger als „das Überleben der Menschheit". Und die Kette der Bedingungen dafür ist schon bekannt: „Kein Überleben ohne Weltethos. Kein Weltfriede ohne Religionsfriede. Kein Religionsfriede ohne Religionsdialog. Kein Religionsdialog ohne Grundlagenforschung. “18

Das Überleben der Menschheit brauche nicht unbedingt an den Weltfrieden gebunden zu werden, meint Spaemann. Ein alles vernichtender Atomkrieg ist kaum wahrscheinlich, viel mehr aber eine Kette von kleineren und größeren Kriegen. Es ist auch nicht ausgemacht, ob eine gewaltige und gewaltsame Ausrottung großer Teile der Menschheit und der Rückfall des Restes in archaische Formen der Zivilisation die Belastung unseres Planeten langfristig nicht erleichtere, also, doch irgendwie dem Überleben der Menschheit diene. ${ }^{19}$

Ebenso fragwürdig sei die Annahme, der Weltfriede hänge von einem Weltethos ab. In der Geschichte haben Menschen und Völker gegeneinander auf Tod und Leben gekämpft, nachdem sie längst durch ein gemeinsames Ethos verbunden waren. Denke man nur an die Religionskriege nach der Reformation. Ein gemeinsames Ethos habe im günstigen Fall zur Humanisierung von Kriegen beigetragen, keineswegs aber zu ihrer Abschaffung. ${ }^{20}$

Nach Spaemann ist es auch nicht unbedingt wahr, dass es kein Weltethos und keinen Weltfrieden ohne Religionsfrieden geben könnte. Es hängt davon ab, wie man Religionsfrieden definiert, trivial oder falsch. Die Behauptung sei trivial, wenn unter Religionsfrieden ein Zustand verstanden wird, in dem Religionen nicht über politische Feind-Freund-Verhältnisse entscheiden, und nicht in der Lage seien, Menschen zu physischer Gewalt gegeneinander zu motivieren. In Konflikten gehe es aber fast nie (nur) um das Religiöse selbst, denke man z. B. an den Libanon und Nordirland. ${ }^{21}$

genden Lebens ist aus einer christlich-theologischen Sicht nicht primär statisch an einem abstrakten Humanum festzumachen, sondern vielmehr in Zeiten erfahrbar und an Orten zu suchen, die Gott selber setzt. Christliche Theologie wird die Erfahrung von Zeiten guten und gelingenden Lebens und von Orten des Friedens und der Verständigung als Erfahrung des Versöhnung stiftenden Handeln Gottes in dieser Welt verstehen."

${ }^{18}$ Robert SpaEmanN, „Weltethos als »Projekt«“", Merkur. Deutsche Zeitschrift für europäisches Denken, 50 (1996), Heft 9/10: 893-904. (894 f.)

${ }^{19}$ A.a.O., 895.

${ }^{20}$ A.a.O., 895 f.

${ }^{21}$ A.a.O., 896 f. 
Auch interreligiöser Dialog kann Kriege nicht unbedingt verhindern. Es gab intensive Religionsdialoge, z. B. vor dem Dreissigjährigen Krieg. Die friedliche Koexistenz und das gemeinchristliche Ethos beruhten nicht auf Religionsdialogen. Sie enthalten, wenn sie ernsthaft sind, immer ein Konfliktpotenzial. „Ein Religionsdialog, der sich als Funktion eines »Ethosprojekts « versteht und unter der Einbildung leidet, der Weltfriede hinge von ihm ab, kann zu nichts führen, was der Rede wert wäre.“222

Grundlagenforschung, oder einfacher gesagt, gründliches, mit Sachkenntnis verbundenes Nachdenken über Religionen ist auch nach Spaemann gewiss wichtig. Aber um sich über das Verbindende klarzuwerden, bedürfe es weder ausgedehnten Nachdenkens, noch institutionalisierter Dialoge. ${ }^{23}$ Es muss doch festgestellt werden: persönliche Begegnungen können mit keinem noch so gründlichen und erfolgreichen Nachdenken langfristig ersetzt werden.

Trotz aller berechtigten Kritik Spaemanns wäre es doch gut, wenn sich immer mehr Menschen das „ins Herz geschriebene Gesetz“ — und seine Grenzen - bewusst machten.

Nach Reinhard Hempelmann hat die Befreiung der westlichen (und füge man hinzu: der orthodoxen) Kultur von der Herrschaft der Religion die Gewalt nicht abgeschafft, noch nicht einmal vermindert, denken wir nur an den Nazionalsozialismus und Stalinismus. Huntington habe einen wichtigen Debattenprozess initiiert, auch wenn er zu Übertreibungen bei der religiösen Deutung von Wirtschaftskonflikten aus ethnisch-kulturellen Aspekten geneigt ist. Er hat uns bewusst gemacht, dass die Religionen eine wichtige Rolle in den politischen Fragen der Zukunft spielen werden. ${ }^{24}$

Was die Erklärung von 1993 angeht, macht Reinhard Hempelmann darauf aufmerksam, dass man die politische Bedeutung der Friedenserklärungen von führenden religiösen Vertretern nicht überschätzen darf. Ein globales Ethos setze mehr oder weniger einen moralischen Enthusiasmus voraus, der den, auf den dieses Ethos gerichtet ist, nicht genug berücksichtigt: den Menschen in seiner Ambivalenz, in seiner Fähigkeit zur Liebe, zum Hass, zur gewaltsamen Durchsetzung seiner Interessen. Niemand kann bestreiten, dass die Religionen grundsätzliche Überzeugungen und Orientierungen an-

\footnotetext{
${ }^{22}$ A.a.O., 897f.

${ }^{23}$ A.a.O., 898.

${ }^{24}$ Reinhard HempelmanN, „Die Prägekraft der Religionen und die Friedensaufgabe der Kirchen“, in Reinhard HempelmanN (Hg.), Religionen und Gewalt, EZW-Texte 167 (Berlin 2002), 59-70. (64.). Er ist nur Namensvetter von Heinzpeter HempelmanN, s. Anm. 12.
} 
bieten, wodurch ethisches Handeln begründet und legitimiert wird. In dem Alltag kommt aber diese Begründung bzw. Legitimierung in dem Maße zur Geltung, in dem es ausdrücklich ein den Alltag bestimmendes religiöses Leben gibt. Das Projekt zieht das nicht genug in Betracht. ${ }^{25}$ Seine Stärke ist aber die Suche nach den Kulturen übergreifenden Prinzipien, vor allem die Berufung auf die Goldene Regel. ${ }^{26}$ — Diese Prinzipien können auch für die der jüdisch-christlichen Kultur entstammenen - Humanisten akzeptabel sein. - Aber nach Hempelmann: „Seine Verbindlichkeit gewinnt ein »Weltethos « allerdings erst im Zusammenhang mit dem Wahrheitsbewusstsein besonderer religiösen Traditionen. Als globales Konzept, das zur Verständigung zwischen Religionen und Kulturen beitragen will, stellt es eher eine Problemanzeige als Problemförderung dar und bleibt hinter seinem Anspruch zurück.“"27

Fassen wir die Kritik aufgrund des obigen Gedankenganges zusammen: Es ist nicht genug, die - wahren oder vermeintlichen - ethischen Prinzipien aus den verschiedenen Religionen „herauszuoperieren“, und sie mit der Kultur des Christentums und der Aufklärung zu „verjüngen“ oder zu „renovieren“. Sie müssen in den Organismus der jeweiligen Religion zurückgepflanzt werden, in der Hoffnung, dass sie nicht „hinausgestoßen“ werden.

\section{DIE NEUEN ATHEISTEN — DIE FRAGE DER ORIENTIERUNG}

Im Rahmen eines einzigen Aufsatzes bleibt nicht genug Raum, die sogenannten Neuen Atheisten und ihre Aktivitäten ausführlich zu behandeln, die betreffende Literatur ist heute fast unübersehbar. ${ }^{28}$ Obwohl die große Aufregung um sie schon vorbei ist, kann man ihre Ansichten und Wirkung nicht einfach abtun und vergessen. ${ }^{29}$ Ich konzentriere mich also par ex-

${ }^{25}$ A.a. O., $64 \mathrm{f}$.

${ }^{26} \mathrm{Ebd}$.

${ }^{27}$ A.a.O., 66.

${ }^{28}$ Eine neue kurze Zusammenfassung s. ANDREAS FinCKE, „Voll im Trend? Anspruch und Wirklichkeit atheistischer, kirchenkritischer und humanistischer Initiativen in Deutschland“, in Matthias Pöhlmann (Hg.), Abschied von der Religion? Säkularisierung - Konfessionslosigkeit - neuer Atheismus, EZW-Texte 257 (Berlin: Evangelische Zentralstelle für Weltanschauungsfragen, 2019, 42-65. Punkt 2. 54-57. „Für Atheisten, Freidenker und Humanisten in Deutschland bedeutete der kometengleiche Aufstieg der neuen Atheisten einen Hoffnungsschimmer.“ Doch diejenigen, die keinen Kontakt mehr mit Kirche und Religion haben wollen, treten zumeist in aller Stille aus. (56 f.)

${ }^{29}$ S. dazu u. a. die gründliche Kritik von Fr. Michael AzKoul, The New Atheists. An Eastern Orthodox Critique, Bloomington, IN: Xlibris, 2017. Er sieht die Möglichkeiten der alten und 
cellence auf zwei von den Vertretern des Atheismus von heute bzw. der jüngsten Vergangenheit, man kann sagen, auf die Prominentesten: Richard Dawkins und der im März 2018 verstorbene Stephen Hawking mit je einem wichtigen Werk. ${ }^{30}$ Fragen und wiederholen wir mit dem Vater des Projektes Weltethos: „Woher kommen denn bestimmte ethische Werte, Maßstäbe und Normen?" ${ }^{\text {31 } ~ „ E s ~ g i b t ~ k e i n ~ V o l k ~ o h n e ~ R e l i g i o n, ~ u n d ~ e r s t ~ r e c h t ~ k e i n ~ V o l k ~ o h n e ~}$ Ethos, das heisst: ohne ganz bestimmte Werte und Maßstäbe.“32 — Woher kommen sie also, und wozu sind sie da, wenn sie als reine Naturprodukte bewertet werden? Aus der Natur, um von uns als reinen Naturprodukten als reine Naturprodukte bewertet werden zu können?

Man kann, besonders als Bürger eines ehemaligen sogenannten sozialistischen Landes die längst ausdiskutierte aber für jede neue Generation immer wieder aktuelle Frage stellen, ob auch der Atheismus eine (Art) Religion sei oder nicht. Als der Marxismus-Leninismus auch in meinem Lande noch als — freilich immer schwächer werdende — „Staatsreligion“ galt, wurde uns an der theologischen Hochschule gelehrt, dass auch der Atheismus eine

neuen Atheisten in der Dekadenz der westlichen Kultur mit ihrer Erkenntnistheorie, also die Übertreibung der Rolle der Vernunft.

${ }^{30}$ Der Begriff kommt von dem amerikanischen Journalisten GARY WOLF, „Battle of the new Atheism“, im Herbst 2006 in Wired Magazine, Stand: 12.01.2019, www.wired.com/wired/ archive/14.11/atheism.htm. Heute: „The Church of the Non-Believers“ https://www.wired.com/ 2006/11/atheism/. Ursprünglich geht es um die „Four horsemen“ (Vgl. Apk 6,1-8): Richard Dawkins (1941), Daniel C. Dennett (1943), Sam Harris (1947) und Christopher Hitchens (19492011). Die Veröffentlichung von Dawkins Buch The God Delusion [Der Gotteswahn, 2006] gilt als Meilenstein in der Geschichte der Neuen Atheisten. S. Die Neuen Atheisten 21.02.2007, Nr. 1211, Stand: 12.01.2019, www.hpd.de/node/1211. Seit der Erscheinung des Buches The Grand Design [Der große Entwurf, 2010] konnte auch Stephen Hawking mehr oder weniger zu ihnen gezählt werden. Now Stephen Hawking joins the New Atheists. Commentary. Originally printed in the $10^{\text {th }}$ September 2010 issue of the Catholic Herald, Stand: 01.09.2013. http://archive. catholicherald.co.uk/article/10th-september-2010/14/now-stephen-hawking-joins-the-new-atheists. Schon 2013 gab es Stimmen, nach denen der neue Atheismus überholt ist, z. B. Ed WEST, „New Atheism is dead“", Catholic Herald, 4 March, 2013, Stand: 15.01.2019, https://catholicherald. co.uk/commentandblogs/2013/03/04/whatever-happened-to-new-atheism/. Theo HoBson, „Richard Dawkins has lost: meet the new atheists“, The Spectator, 13, April 2013, Stand: 15.01.2019. https://www.spectator.co.uk/2013/04/after-the-new-atheism/.

${ }^{31}$ Hans KüNG, Der Anfang aller Dinge (München, Zürich: Piper, 2006), 211. In diesem Punkt zu Hawkings Ausführungen vgl. Péter SzENTPÉTERY, „Trónfosztás — vagy csak behunyt szemmel a trón előtt? Hozzászólás Brendel Mátyás írásához“ [Entthronung — oder nur mit geschlossenen Augen vor dem Thron? Anmerkungen zum Artikel von Mátyás Brendel], Magyar Tudomány [Ungarische Wissenschaft, Monattsschrift der Ungarischen Akademie der Wissenschaften] 10 (2012): 1248-1251., s. dazu: Mátyás BRENDEL, A nagy trónfosztás [Die grosse Entthronung] A.a.O. 3 (2012): 349-353.

${ }^{32}$ KÜNG, Anfang (s. Anm. 32) 212. 
Religion ist: warum den leugnen, den es sonst nicht gibt? Also, auch der Atheist muss sich irgendeinen Gott vorstellen, oder genauer gesagt, ein Gottesbild malen, von dem fesgestellt werden kann, dass er/es nicht existiert. Von Reinhard Hempelmann wird es treffend formuliert: „A-Theismus ist ein Relationsbegriff. Er bezieht sich auf wie auch immer geartete Vorstellungen von Gott, die verneint werden. Insofern gibt es den Atheismus nicht ohne den Gottesglauben. Und es kann so viele Formen des Atheismus geben, wie es Ausdruckformen des Glaubens gibt. ${ }^{\text {“33 }}$ Aber die Grundprinzipien des Weltethos gelten für alle Religionen, logischerweise auch für diejenigen, die sich einer Leugnung der Existenz Gottes als Grundlage bedienen.

Man muss Paul Tillich Recht geben: die Behauptung dass „Gott existiert“ kann auch als ungewollte Blasphemie bezeichnet werden, weil sie Gott so beschreibt, als ob er den Kategorien von Existenz und Nichtexistenz unterstellt werden könnte. Gott ist nicht einer der Existierenden, auch nicht als der Größte aller Existierenden, sondern der Urgrund, die Grundstruktur aller Existenz. ${ }^{34}$ Wenn Gott aber von Atheisten nur als Lückenbüßer noch nicht erklärter naturwissenschaftlicher Probleme betrachtet wird, gelte dasselbe auch für bestimmte ethische Werte, Maßstäbe und Normen.

Hawking, der auch Mitglied der Päpstlichen Akademie der Wissenschaften war, ruft in seinem ganzen Buch: Der große Entwurf zur Entscheidung zwischen Gott und den Gesetzen der Physik auf. Wenn Küng sagt, dass nur Unbedingtes unbedingt verpflichten kann, lautet die Frage an Hawking \& Co., wozu, zu welchem Ethos uns die Gesetze der Physik verpflichten könn(t)en.

Im Projekt Weltethos geht es also um eine gemeinsame Konkretisierung unterschiedlich begründeter (oder manchmal —, wie in diesem Fall — quasi begründeter) Orientierungen. Diese Orientierungen an sich reichen aber als konkrete Standards noch nicht aus. Im Falle eines angeblich naturwissenschaftlichen Atheismus diene dann die Befreiung des Menschen vom Joch der Religion dem echten Humanum als Begründung wie das bei Dawkins der Fall ist. Auch für die Neuen Atheisten gilt, was Hans Küng feststellt: „Das Christentum ist nach wie vor für einen Großteil der westlichen Natur-

\footnotetext{
${ }^{33}$ Reinhard Hempelmann, „Der Neue Atheismus hat verschiedene Facetten. Vision einer religionsfreien Welt", Herder Korrespondenz 2014, 1: 2-5. 2. Man denke darüber hinaus an Personifikationen, Symbole, Riten usw. z. B. die Vernunftsgöttin der französischen Revolution, die Religion und der Tempel der Vernunft bei August Comte oder Ernst A. F. Haeckel als Gegenpapst.

${ }^{34}$ Vgl. Paul Tillich, Systematische Theologie, Bd. I (Stuttgart: Evangelisches Verlagswerk, 21956), 203.
} 
wissenschaftler der implizite weltanschauliche Hintergrund [...]. ${ }^{\text {35 }}$ Dawkins strebt nach einer idealen christlichen Sittlichkeit, abgeschnitten von den christlichen Wurzeln. Das selbstsüchtige Gen und der blinde Uhrmacher als (die) Unbedingte(n) scheinen ihn dazu zu verpflichten. ${ }^{36}$

Hawking will Gott dadurch unnötig machen, dass er die Entstehung der Welt auf die Gravitation zurückführt. Die Existenz der Gravitation bedeutet für ihn, dass die Entstehung des Universums unvermeidbar war. So konnte und kann sich das Universum aus dem Nichts schaffen. (Gravitation ist doch mehr als nichts!) Das Multiversum-Konzept könne die Feinabstimmung der physikalischen Gesetze erklären, ohne einen gütigen [und gültigen] Schöpfer einbeziehen zu müssen, der das (einzige) Universum zu unserem Nutzen erschuf. ${ }^{37}$ Die eigentliche Frage ist, was Hawking gleich am Anfang des Buches aufwirft und gegen Ende wiederholt: „[...] der Mensch ist ein neugieriges Spezies. Wir staunen und suchen nach Antworten. “38 Und am Ende des ersten Kapitels: „Warum gibt es etwas und nicht einfach nichts?“ Also die Grundfrage der Philosophie nach Leibniz. - Die zwei weiteren, auch auf Leibniz' Spur, folgen daraus: „Warum existieren wir? Warum dieses besondere System von Gesetzen und nicht irgendein anderes?“39 Und gegen Ende des Buches: „Wie können wir entscheiden, ob ein Wesen einen freien Willen besitzt? Wie können wir entscheiden, ob wir mit einem Roboter oder einem intelligenten Geschöpf zu tun haben, wenn wir einem Außerirdischen begegneten?" Bei einem so komplizierten Außerirdischen wie einem Menschen, sagt er, wäre uns die Berechnung, die Vorhersage des Benehmens aller seinen Teilchen zu kompliziert, dies zu entscheiden. Also, man müsste sagen, dass er aller Wahrscheinlichkeit nach einen freien Willen hat. Damit wäre keine fundamentale Eigenschaft postuliert, sondern eine praktische Theorie formuliert. Es wird einfach eingestanden, dass wir die nötigen Vorhersagen zu seinen Handlungen nicht ausrechnen können. ${ }^{40}$

Also, auch dem Menschen muss freier Wille postuliert werden, einfach dadurch, dass der Mensch zu kompliziert ist, alle seinen Handlungen im

\footnotetext{
35 KüNG, Anfang (s. Anm. 33), 215.

${ }^{36}$ Die zwei Werke, die Dawkins weltweit bekannt gemacht haben (The Selfish Gene, 1976; The Blind Watchmaker, 1986). Zugespitzt könnte man so formulieren, dass das erstere als das Evangelium und das letztere als der Römerbrief für die Bewegung des Neuen Atheismus gilt.

${ }^{37}$ Stephen HawKING und Leonard MLODINOw, Der Große Entwurf. Eine neue Erklärung des Universums (Berlin: Nicolai-Verlag, 2010), 163., s. Kap 7, 147 ff.

${ }^{38}$ A.a.O., 11

${ }^{39}$ A.a.O., 15., vgl. 167.

${ }^{40}$ A.a.O., 175.
} 
Voraus berechnen zu können. Freiheit stamme freilich aus Nicht-Freiheit, weil die Entstehung des Universums/der Universen eine Notwendigkeit sei. Wozu aber freier Wille, freies Ethos als Folge komplizierter Organisierung der Elementarteilchen? Bin ich keinesfalls frei, wenigstens nicht in dem Maße, dass ich über die Möglichkeit von Freiheit und Nicht-Freiheit überhaupt Gedanken machen kann?

Hawkings Schlussfolgerung ist einfach enttäuschend: „Die Tatsache, dass wir - Menschen, die wir selbst nur eine Ansammlung fundamentaler Naturteilchen sind - dem Verständnis der Gesetze, die uns und unser Universum regieren, so nahe gekommen sind, ist ein großer Triumph. “41 Die Frage ist dann „nur“, ob wir aus freiem Willen darauf gekommen sind, dass das Universum, das uns enthält, aus dem Nichts entstanden ist. Wir „wissen“, dass wir nur eine Ansammlung fundamentaler Naturteilchen sind, aber kompliziert genug, aus freiem (in seinem Gedankenrahmen freilich aus unfreiem) Willen Ja z. B. zum Weltethos zu sagen. Im dem Buch umgeht er das eigentliche Problem, er nimmt als gegeben, dass der Mensch ein staunendes und fragendes Wesen ist, der - sei es aus freiem oder unfreiem Willen - über sich hinausgehen bzw. sich immer überbieten will. Er stellt also nicht die Frage, warum der Mensch, (auch er) zur Unendlichkeit hin offen ist. Behauptungen des ontologischen Reduktionismus wie „nur das und das“, „nichts anderes als“, „nichts mehr als“ sind keine naturwissenschaftlichen Aussagen ${ }^{42}$ sondern - unbeabsichtigt — verweisen auf die Offenheit des Menschen zum Unendlichen. Er und andere „wissenschaftliche“ Atheisten möchten aber auf die konsequente Erforschung dieser Offenheit aus welchen Gründen auch immer lieber verzichten. Auch Hawking war und seine Mitstreiter sind offen zur Unendlichkeit hin, wenn sie viele-viele Universen vermuten, um an keinen (persönlichen) Schöpfer glauben zu müssen. Das ist aber keine Neuheit, wir wissen, dass z. B. schon Epikur und Lucretius etwas Ähnliches erfunden hatten. ${ }^{43}$

Richard Dawkins will auf seine Weise eine Ethik und damit ein Weltethos ohne Gott begründen, das die Ethik der Religionen weit übertreffen kann. Er

\footnotetext{
${ }^{41}$ Hawking, Der Große Entwurf (s. Anm. 41), 177.

${ }^{42}$ John LENNOX, Hat die Wissenschaft Gott begraben? Eine kritische Analyse moderner Denkvoraussetzungen (Wuppertal: Brockhaus, 2001), 36. Am Anfang des Buches schreibt er, Dawkins hatte vielleicht das Pech, zu vielen antiwissenschaftlichen und aufklärungsfeindlichen Christen zu begegnen. $5 \mathrm{f}$.

${ }^{43}$ Zur Offenheit des Menschen zum Unendlichen hin s. das bekannte kurze Grundlagewerk von Wolfhart PANNEnBerg, Was ist der Mensch? Die Anthropologie der Gegenwart im Lichte der Theologie (Göttingen: Vandenhoeck \& Ruprecht, ${ }^{7} 1985$ ).
} 
verwirft das Alte Testament mit allen seinen grausamen Geschichten: z. B. Sodoma und Gomorrha, die Landnahme von Josua usw. ${ }^{44}$ Jesus bedeute aber einen großen Fortschritt, und er gelte als einer der bedeutendsten ethischen Erneuerer der Geschichte. Die Lehre von der Erbsünde bleibe jedoch nicht weit hinter den Grausamkeiten des Alten Testaments. Warum sind diejenigen $\mathrm{zu}$ bestrafen, die noch keine Verbrechen begehen konnten, und warum sind sie von deren Folgen durch die Sünde derer zu erlösen, die den Erlöser geschlagen und hingerichtet hatten ${ }^{45}$ Religionen seien für viele Greueltaten verantwortlich, der Atheismus aber nicht. Er beleuchtet das am Beispiel von Hitler und Stalin. Stalin war Atheist, Hitler aber trotz anderen Behauptungen aller Wahrscheinlichkeit nach nicht. Atheismus sei doch viel ethischer als Religion. Einige Atheisten begehen böse Taten, sie tun dies aber nicht im Namen des Atheismus. „Stalin und Hitler begingen entsetzliche Taten, der eine im Namen eines dogmatischen, doktrinären Marxismus, der andere im Namen einer krankhaften, unwissenschaftlichen Theorie der Erbgesundheit, die mit halb-wagnerianischen Fantastereien unterlegt war." Religionskriege wurden offensichtlich im Namen der Religion geführt, Dawkins wisse aber von keinem Krieg, der im Zeichen des Atheismus geführt worden wäre. ${ }^{46}$ Er rechnet offensichtlich nicht damit, dass Religion auf den ganzen Menschen auswirkt, nicht nur entweder auf seine Vernunft oder auf seine Gefühle. Dasselbe muss natürlich für Religionslosigkeit bzw. für negative Religion gelten. Der Atheismus kann im grausamen Lebenslauf des ehemaligen orthodoxen Seminaristen nicht isoliert betrachtet werden, abgesehen davon, dass während seiner Terrorherrschaft abertausende Kirchen zerstört, gesperrt oder umfunktioniert wurden, und atheistische Propaganda Hand in Hand mit seinem Personenkult mit Blut und Tränen verbreitet wurde. Es ist kein entscheidendes Argument, dass man nicht unbedingt religiös sein muss, um hohen ethischen Standards entsprechen $\mathrm{zu}$ können. Die Frage bleibt nach wie vor, woher die sittliche Orientierung des Menschen überhaupt kommt, ob Freiheit aus Nicht-Freiheit entstehen kann.

\footnotetext{
${ }^{44}$ Richard DawKINS, Der Gotteswahn (Berlin: Ullstein, $\left.{ }^{10} 2007\right), 331$ f., 342.

${ }^{45}$ A.a.O., 266 ff. Der Gotteswahn wurde sofort und als Grundlagewerk des Neuen Atheismus betrachtet und (heftig) diskutiert. Die bekannteste Antwort kurz danach kam von Oxforder Biochemiker, Theologe und früherem Atheisten Alister McGrath (mit Joanna Collicutt McGrath), The Dawkins Delusion. Atheist fundamentalism and the denial of the divine (London: InterVarsity Press, 2007) (Alister McGrath und Joanna Collicutt McGrath, Der Atheismus-Wahn. Eine Antwort auf Richard Dawkins und den Atheistischen Fundamentalismus (Asslar: GerthMedien, $\left.{ }^{2} 2008\right)$.

${ }^{46}$ A.a.O., 378 ff., bes. 387.
} 
Küng ist der Meinung, dass in gegenseitigem Respekt eine Koalition von Glaubenden und Nichtglaubenden (Deisten, Atheisten, Agnostiker) zugunsten eines gemeinsamen Weltethos notwendig sei. Was die Realisierbarkeit einer solchen Koalition angeht, sagt er: „[...] mit den Glaubenden können auch Nichtglaubende Widerstand üben gegen allen trivialen Nihilismus, gegen diffusen Zynismus und soziale Kälte und sich überzeugt und überzeugend für die Grundrechte aller Menschen, für die Verkleinerung der Kluft zwischen Reichen und Armen, für den Halt des Zuwaches der Slums in der Vierten Welt, für die Verhinderung des Wohlstandsniveaus durch Ökokatastrophen und internationale Wanderbewegungen, und für eine kriegslose Weltgesellschaft einsetzen.“47

Auch im Falle von Dawkins, wie schon oben erwähnt, ergibt sich unbedingt die Frage: Was ist das Unbedingte, das unbedingt verpflichten kann? Wie passt jemand in dieses Schema, der Religion als solche ,virus of the mind“48 nennt, Er kämpft gegen Religion als solche, er kann also auch diejenigen Theologen nicht als Mitstreiter akzeptieren, die sonst das heutige kosmologisch-evolutionistische Weltbild mit ihm teilen. ${ }^{49}$

Dawkins stellt eine 7-gradige Skala zwischen Theismus und Atheismus auf, und sagt, dass es nur sehr wenige gibt, die Stufe 7 erreichen. Er gesteht ein - das sei zu seinem Lob gesagt - , er sei nur auf Stufe 6, also: nach ihm, für ihn gibt es wahrscheinlich keinen Gott. ${ }^{50}$ Will er mit den vielen Schlachtrufen und falschen Behauptungen seine nicht hundertprozentig versteckten und verdrängten Zweifel immer wieder übertönen? Was ist der spezielle Beitrag des Dialogs zwischen Christentum und kämpferischem Atheismus zum Weltfrieden?

\section{ZUSAMMENFASSUNG UND THESEN}

Von christlicher Identität wurde beabsichtigt nur zwischen den Zeilen gesprochen. Niemand kann die Gemeinsamkeiten in der ethischen Orientierung der Religionen bestreiten, auch Paulus war sich nach Apg 17,22 ff.

\footnotetext{
${ }^{47}$ KüNG, Projekt Weltethos (s. Anm. 4), $58 \mathrm{ff}$.

${ }^{48}$ Dawkins, Der Gotteswahhn (s. Anm. 47) 260 ff., 275.

${ }^{49}$ A.a.O., 95ff. Trotz seiner gegnerischen Ansichten über Christentum und Religion debattierte Dawkins ganz locker und gemütlich mit hochrangigen kirchlichen Vertretern und anderen Gesprächpartnern.

${ }^{50}$ Dawkins, Der Gotteswahn, $72 \mathrm{f}$.
} 
und Röm 2,12 ff. darüber im Klaren. Es ist also nichts Neues, wenn seit einer Zeit immer nachdrücklicher darauf hingewiesen wird. Die Allgemeinheit der Goldenen Regel bestätigt das, für uns als Christen gilt vor allem ihre positive Formulierung (Matth. 7,12).

1. Die Basis des Weltethos ist das Humanum. Dieser Begriff ist aber auf dem Boden der jüdisch-christlichen Kultur und der aus dieser Kultur gewachsenen Aufklärung entstanden. Freiheit, Gleichheit, Brüderlichkeit und Menschenwürde sind ursprünglich christliche Werte. Es gehört zu unserer christlichen Identität, das offenzulegen.

2. Trotz aller berechtigten Kritik am Weltethos-Projekt is festzustellen: es ist empfehlenswert und notwendig, sich dieses ins Herzen geschriebene Gesetz immer wieder bewusst zu machen. Umso mehr, weil Gott es so gemacht hat, dass von Einem aller Menschen Geschlechter stammen.

3. Menschen werden aller Wahrscheinlichkeit nach zum ethischen Handeln dadurch kaum besser motiviert, wenn sie entdecken, dass ähnliche Motivationen auch bei Vertretern anderer Religionen vorkommen. Motivation muss aus der eigenen Überzeugung/Religion kommen. Persönliche Kontakte können doch nicht ausbleiben, und im Alltag des „Weltdorfes“ kommen sie sowieso immer öfter vor.

4. Kritik am Weltethos-Projekt ist berechtigt, indem seine Wirkung nicht übertrieben werden soll, sondern in aller Nüchternheit eher kleine Schritte von ihm erwartet werden können. - Dialog dient eher zur Konfliktvermeidung als zur Problemlösung.

5. Das Weltethos will eher ein ethisches als ein religiöses Projekt sein. Es hängt aber davon ab, wie man Religion, Ersatzreligion, negative Religion bzw. Theologie definiert.

Eine Koalition von Glaubenden und Nichtglaubenden (Deisten, Atheisten, Agnostiker) in gegenseitigem Respekt zugunsten eines gemeinsamen Weltethos wäre nach dem Projekt notwendig. Also, um des ethischen Handelns willen wird beiseite gelegt, wie dieses Handeln begründet wird.

6. Vom Standpunkt der christlichen Identität aus ist es immer eine Frage, wie ein Christ mit Vertretern von ausdrücklichen Irrlehren umgeht. Das noch so schöne Ziel des Weltfriedens heiligt nicht unbedingt die falschen Begründungen als Mittel zu seinem Erreichen.

7. Indem nur Unbedingtes unbedingt verpflichten kann, ergibt sich die Frage aufgrund Stephen Hawkings Erörterungen, wie man über freien Willen denkt. Wenn freier Willen nur aufgrund der Kompliziertheit eines vernünftigen Wesens postuliert werden kann, wird Freiheit mit der Nicht-Freiheit (des 
Bestimmtseins durch äußere Gegebenheiten) erklärt? Sittliches Handeln ist doch möglich, „nur“ seine Begründung hängt im Falle von Hawking (und vielen „wissenschaftlichen“ Atheisten) in der Luft.

8. Eine weitere Frage ist, wie diejenigen in das Schema der WeltethosKoalition passen, die Religion als solche für schädlich halten, z. B. Richard Dawkins. Doch ist bei ihm ernst zu nehmen, dass er die Christen dafür zur Rechenschaft zieht, dass sie ihrem Meister seit fast zwei Jahrtausenden nur so wenig gehorchen. Christliche Identität kann und muss auch von den alten und neuen - Atheisten gestärkt werden.

Zum Schluss: Christliche Identität bedeutet, dass Christen sich mit ihrem Meister immer mehr identifizieren, d. h., ihm nachfolgen wollen. Für uns ist Christus der Maßstab, die Begründung, die Orientierungshilfe zum ethischen Handeln, also zu seiner Nachfolge, zu ihm selber.

\section{BIBLIOGRAPHIE}

Azkoul, Michael. The New Atheists. An Eastern Orthodox Critique. Bloomington, IN: Xlibris, 2017.

BAuschKe, Jürgen. Die Goldene Regel. Staunen - Verstehen - Handeln. Berlin: EB-Verlag, 2010.

DAwkINS, Richard. Der Gotteswahn. Berlin: Ullstein, ${ }^{10} 2007$.

FINCKE, Andreas. „Voll im Trend? Anspruch und Wirklichkeit atheistischer, kirchenkritischer und humanistischer Initiativen in Deutschland“. In Abschied von der Religion? Säkularisierung - Konfessionslosigkeit - neuer Atheismus. EZW-Texte 257, herausgegeben von Matthias Pöhlmann, 42-65. Berlin: Evangelische Zentralstelle für Weltanschauungsfragen, 2019.

Hawking, Stephen, und Leonard Mlodinow. Der Große Entwurf. Eine neue Erklärung des Universums. Berlin: Nicolai-Verlag, 2010

HempelmanN, Heinzpeter. „Intolerante Toleranz. Hans Küngs »Projekt Weltethos« als Prokrustesbett religiöser Geltungsansprüche." Theologische Beiträge 33, Febr. 2002. Stand: 11.01.2019. https://heinzpeter-hempelmann.de/hph/wp-content/uploads/2013/01/ kueng.pdf.

Hempelmann, Reinhard. „Der Neue Atheismus hat verschiedene Facetten. Vision einer religionsfreien Welt". Herder Korrespondenz 2014, 1: 2-5.

Hempelmann, Reinhard. „Die Prägekraft der Religionen und die Friedensaufgabe der Kirchen“. In Religionen und Gewalt. EZW-Texte 167, herausgegeben von Reinhard Hempelmann und Johannes Kandel, 59-70. Berlin: Evangelische Zentralstelle für Weltanschauungsfragen, 2002.

KüNG, Hans (Hg.). Dokumentation zum Weltethos. München, Zürich: Piper, 2002. 
KüNG, Hans (Hg.). Ja zum Weltethos. Perspektiven für die Suche nach Orientierung. München: Piper, 1995.

KÜNG, Hans, und Angela RINN-MAurer. Weltethos christlich verstanden. Positionen, Erfahrungen, Impulse. Freiburg, Basel, Wien: Herder, 2009.

KÜNG, Hans. „Dialog der Kulturen“, 9. November 2001 [also, wie er selber betont, nicht lange nach dem 11. September], Stand: 11.01.2019, https://www.weltethos.org/1-pdf/20aktivitaeten/ deu/politik/un-statement-Kueng.pdf.

KÜNG, Hans. Christ sein. München: Dt. Taschenbuch-Verlag ${ }^{8} 1987$.

KÜNG, Hans. Christentum und Weltreligionen. München: Herder, 1984.

KüNG, Hans. Der Anfang aller Dinge. München, Zürich: Piper, 2006.

KÜNG, Hans. Projekt Weltethos, München: Piper, 1990.

KüNG, Hans. Spurensuche. Die Weltreligionen auf dem Weg, München, Zürich: Piper, ${ }^{4} 2000$.

KüNG, Hans. Was ich glaube. München, Zürich: Piper, 2010.

KÜNG, Hans. Weltethos für Weltpolitik und Weltwirtschaft. München, Zürich: Piper, ${ }^{3} 1998$.

LENNOX, John. Hat die Wissenschaft Gott begraben? Eine kritische Analyse moderner Denkvoraussetzungen. Wuppertal: Brockhaus, 2001.

Manifesto, Global Economic Ethic, Consequencen for Global Businesses. UN Headquarters, New York, 6 October 2009. Stand: 11.01.2019. www.globaleconomicethic.org/main/pdf/ ENG/ we-manifest.ENG.pdf.

McGrath, Alister, und Joanna Collicutt McGrath. Der Atheismus-Wahn. Eine Antwort auf Richard Dawkins und den Atheistischen Fundamentalismus. Asslar: GerthMedien, ${ }^{2} 2008$.

PANNENBERG, Wolfhart. Was ist der Mensch? Die Anthropologie der Gegenwart im Lichte der Theologie. Göttingen: Vandenhoeck \& Ruprecht, ${ }^{7} 1985$.

REHM, Johannes. Weltethos praktisch. Perspektiven für den interreligiösen Dialog. Mainz: Matthias-Grünewald-Verlag, 2004.

Spaemann, Robert. „Weltethos als »Projekt«““. Merkur. Deutsche Zeitschrift für europäisches Denken, 50 (1996), Heft 9/10: 893-904.

SzentPÉTERY, Péter. „Trónfosztás — vagy csak behunyt szemmel a trón előtt? Hozzászólás Brendel Mátyás írásához“ [Entthronung - oder nur mit geschlossenen Augen vor dem Thron? Anmerkungen zum Artikel von Mátyás Brendel]. Magyar Tudomány [Ungarische Wissenschaft, Monattsschrift der Ungarischen Akademie der Wissenschaften] 10 (2012): $1248-1251$.

SzENTPÉTERY, Péter, und András SzALAI, „Világetosz, párbeszéd, egyezkedő szó [Weltethos, Dia$\log$, und gemeinsames Wort]“. Vallástudományi Szemle [Religionswissenschaftliche Schau, Vierteljahresschrift der Ungarischen Gesellschaft für Religionswissenschaft] 2 (2011): $11-25$.

TILLICH, Paul. Systematische Theologie. Bd. I. Stuttgart: Evangelisches Verlagswerk ${ }^{2} 1956,203$. Weltethos-Institut. Stand: 11. 01. 2019. http://www.weltethos-institut.org/institut/.

\section{PROJEKT ETYKI GLOBALNEJ (WELTETHOS), NOWY ATEIZM I TOŻSAMOŚĆ CHRZEŚCIJAŃSKA}

$$
\text { Streszczenie }
$$

Projekt Etyki Globalnej jest ściśle związany z jego twórcą, Hansem Küngiem (1928). Opiera się na fakcie, że w różnych religiach występują podobne nakazy etyczne, które mogą być ujmowane jako tzw. Złota Reguła. Pierwsza część artykułu wprowadza w projekt i stanowi jego krytykę, przede wszystkim dlatego, że rozciaga on pochodzące z zachodniego chrześcijaństwa pojęcie ludzkości również na inne religie. Druga część artykułu ukazuje, że Hans Küng chce 
angażować nie tylko ludzi wierzących, ale również ateistów, agnostyków itd., jako że Projekt Etyki Globalnej ma przede wszystkim charakter etyczny. To dlatego autor koncentruje się na dwóch dobrze znanych dziełach prominentnych przedstawicieli tzw. Nowego Ateizmu, aby spróbować odpowiedzieć na pytanie, do jakiego stopnia ich twierdzenia zbiegają się z Projektem. $\mathrm{W}$ podsumowaniu formułuje konkluzję składającą się z ośmiu tez.

Słowa kluczowe: Etyka Globalna; Hans Küng; religia; dialog; ludzkość; Nowy Atezim; Stephen Hawking; Richard Dawkins. 